\title{
MODELO INTELIGENTE GENERICO PARA ADAPTATIVIDAD DE CURSOS VIRTUALES
}

\author{
Néstor Darío Duque Méndez, $\mathrm{PhD}$ (c) \\ Universidad Nacional de Colombia, \\ Depto de Informática y Computación, \\ Sede Manizales, Colombia \\ ndduqueme@unal.edu.co \\ Demetrio Ovalle Carranza, $\mathrm{PhD}$ \\ Universidad Nacional de Colombia, \\ Escuela de Sistemas, \\ Sede Medellín, Colombia \\ dovalle@unalmed.edu.co \\ Rosa Vicari, $\mathrm{PhD}$ \\ Universidad Federal de Rio Grande del Sur UFRGS, Brasil, \\ rosa@inf.ufrgs.br \\ Ricardo Azambuja Silveira, $\mathrm{PhD}$ \\ Universidad Federal de Santa Catarina UFSC, Brasil, \\ silveira@inf.ufsc.br
}

\section{Resumen}

El presente articulo expone una propuesta genérico para cursos virtuales adaptativos soportado en técnicas de inteligencia artificial, en particular sistemas multi-agente, planificación en inteligencia artificial y razonamiento basado en casos. La implementación mediante un sistema multiagente y la definición de un framework para especificar la estrategia de adaptación permite incorporar variados enfoques pedagógicos y tecnológicos, según las visiones del equipo en la instalación concreta.

La generación automática entrega un curso personalizado aplicando una explicita estrategia de adaptación que reconoce diversas características de cada aprendiz (psicológicas, psicopedagógicas, preferencias y los logros obtenidos, expresados en términos de Objetivos Educativos).

Palabras Claves: Educación Virtual, sistemas adaptativos, inteligencia artificial en educación

\begin{abstract}
This work presents/displays an adaptive generic model for virtual courses supported in techniques of artificial intelligence, in individual systems multi-agent, planning in artificial intelligence and reasoning based on cases. The automatic generation gives a customized course applying one specifies adaptation strategy that recognizes diverse characteristics of each apprentice (psychological, psicopedagógicas, the obtained, expressed preferences
\end{abstract}


and profits in terms of Educative Objectives). The implementation by means of a system multiagent and the definition of framework to specify the adaptation strategy allow to incorporate varied pedagogical and technological approaches, according to the visions of the equipment in the concrete installation.

\section{Introducción}

La adaptabilidad del sistema puede entenderse como la capacidad del sistema para que dinámicamente adapte su conducta a los requerimientos de la interacción usuario-sistema (Duque, 2007).

Las expectativas, expresadas desde los años 70, de una educación individualizada, apoyada en, por ese entonces, Tecnologías de la Información y la Comunicación (TIC), donde se reconociera a cada estudiante en particular, omitiera instrucción hacia logros ya obtenidos, respete el ritmo de avance del aprendiz, posibilite diferentes caminos hacia el objetivo educativo propuesto con ricos materiales y actividades multimodales (Eraut, 1970; Stolurow,1970; Alfaro et al., 1998), aun sigue siendo una meta. Pero ante tantas promesas y esperanzas acerca de la revolución e-learning y con todos los avances en tecnologías multimedia, un cerrado escrutinio permite revelar que muchos de estos "modernos" sistemas, son poco más que el viejo aprendizaje apoyado por computador basado en texto pero ahora corriendo en Internet (Dastbaz et al., 2006).

La comunidad en este campo avanza por diferentes caminos en busca de hacer realidad las promesas y esperanzas generadas y la revisión de las diferentes tendencias en sistemas de educación virtual colocan a los sistemas adaptativos y a los sistemas pedagógicos inteligentes como temas de vanguardia en las investigaciones y propuestas (ITS, 2006; ED-MEDIA, 2006).

Una real y profunda personalización requiere precisar una estrategia de adaptación, lo que implica definir los elementos a adaptar, las características que determinan cuándo adaptar y una serie de reglas que gestionen el proceso. En el caso particular de los sistemas educativos esta estrategia debe además recoger el clamor de la comunidad internacional expresado en The 3rd workshop of Authoring of Adaptive and Adaptable Educational Hypermedia en Holanda en el 2005 (Cristea et al., 2005) en el sentido de determinar cuáles son las principales características a modelar en el estudiante, definir cómo formular el conocimiento pedagógico de una manera reusable soportando escenarios pedagógicos y considerando los estilos cognitivos.

Este artículo propone un nuevo género de cursos personalizados soportados en una estrategia genérica de adaptación y modelados a través de Sistemas Muti-Agente utilizando técnicas de Inteligencia Artificial. También expone el análisis realizado y los resultados obtenidos que permiten la definición clara de una estrategia de adaptación para la generación automática de un curso personalizado, basado en la especificación de determinantes y componentes que soportados en metadatos faciliten el proceso.

El resto del material está organizado así: el numeral siguiente recoge algunos conceptos de los sistemas adaptativos, el apartado 3 presenta la propuesta conceptualmente, para continuar con la exposición del proceso de construcción del sistema multiagente y finaliza con las conclusiones y trabajos futuros.

\section{Educación Personalizada}


Como se aprecia en la figura 1 los componentes susceptibles de adaptabilidad en un sistema de educación pueden ser las interfases con el estudiante, el plan del curso, las estrategias educativas, el filtrado de información y el proceso evaluativo, integrando actividades individuales con ambientes colaborativos.

La adaptación debe realizarse mediante una determinada estrategia de adaptación, lo que implica definir los aspectos a adaptar, las condiciones para esa adaptación, los objetivos que se persiguen y la forma en que se realizará, en otras palabras, Qué Adaptar, Cuando adaptar, las Metas de Adaptabilidad y las reglas de adaptabilidad (Karagiannidis et al., 1996); o delimitar un contexto de referencia que determine el ámbito de la conducta de adaptación y el orden parcial de los casos de adaptación y define dos componentes principales: Un conjunto de disparadores de adaptación y un conjunto de casos de adaptación asociados con los disparadores (Paques et al., 2004).

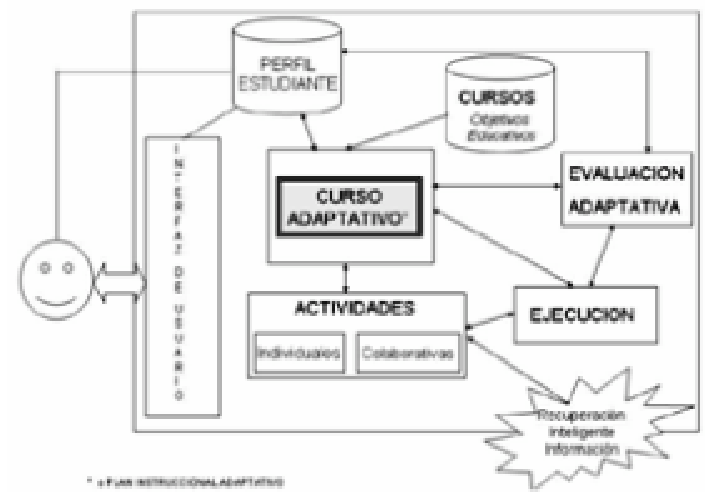

Figura 1. Relación de elementos en un sistema de cursos adaptativos

Las tecnologías de adaptación de sistemas en Web pueden resumirse en selección adaptativa de contenidos, soporte de navegación adaptativa y presentación adaptativa (Brusilovsky y Maybury, 2002). Duque et Al. (2006) refieren que la adaptación también puede verse en otros momentos del proceso educativo y que las técnicas pueden orientarse a secuencia del currículo, soporte adaptativo a tareas colaborativas, al análisis inteligente de soluciones, a la recuperación inteligente de información y a la evaluación personalizada (Duque et al., 2006).

\section{Sistema adaptativo para generación de cursos.}

Proponer un sistema adaptativo en sistemas educativos requiere la definición precisa de varios componentes de tal forma que permitan su manipulación en el proceso de adaptación.

Como se aprecia la tarea de adaptación debe definir, por un lado, los elementos relevantes del perfil del estudiante que determinan la personalización y por otro lado, el dominio del curso debe ser representado de tal forma que pueda ser adaptado según las necesidades de los aprendices, especificando los componentes susceptibles de adaptación, según el enfoque del sistema. La automatización de este proceso requiere y exige una estrategia clara de adaptación que conjugue estos elementos mediante reglas o algoritmos que ponderando las necesidades y metas expresadas entregue un curso personalizado a cada estudiante.

Enfrentar este problema implica aunar dos esfuerzos, por un lado la pedagogía y tecnologías educativas y por el otro las tecnologías informáticas que diseñen $\mathrm{y}$ 
construyan los sistemas que soporten las expectativas de personalización. En este punto las técnicas de inteligencia artificial han mostrado sus bondades, entre ellas la aplicación de razonamiento basado en casos (RBC), redes neuronales $(\mathrm{NN})$, planificación inteligente (IA Planning), redes bayesianas (RB), sistemas fuzzy y algoritmos genéticos (AG). En varios de los trabajos revisados hay un enfoque apoyado en Sistemas de Agentes Inteligentes (SMA), que parece ser una promisoria alternativa (Jaques y Vicari, 2005; Jimenez, 2006; VIcari, 2005; Merida y Fabregat, 2003; Martins y Diniz, 2004; Nakagawa y Kuroda, 2004, Silveira, 2001).

La figura 2 presenta mediante un mapa conceptual las diversas propuestas y tecnologías encontradas en la revisión realizada e integra conceptos y acercamientos a las técnicas a emplear.

La propuesta que se plantea es expuesta en la figura 3. Se aprecia que existe una separación entre la estructura del curso (representada en objetivos educativos o competencias a conseguir) y los materiales educativos que apoyan las actividades educativas según estrategias pedagógicas diversas, aplicadas a las diferencias de los aprendices. Para el estudiante se definen las características más relevantes dentro del proceso educativo tanto a nivel psicológico, psicopedagógico, las no permanentes y algunas de tipo contextual tecnológico. Su perfil académico se almacena en términos de los objetivos educativos que ha logrado.

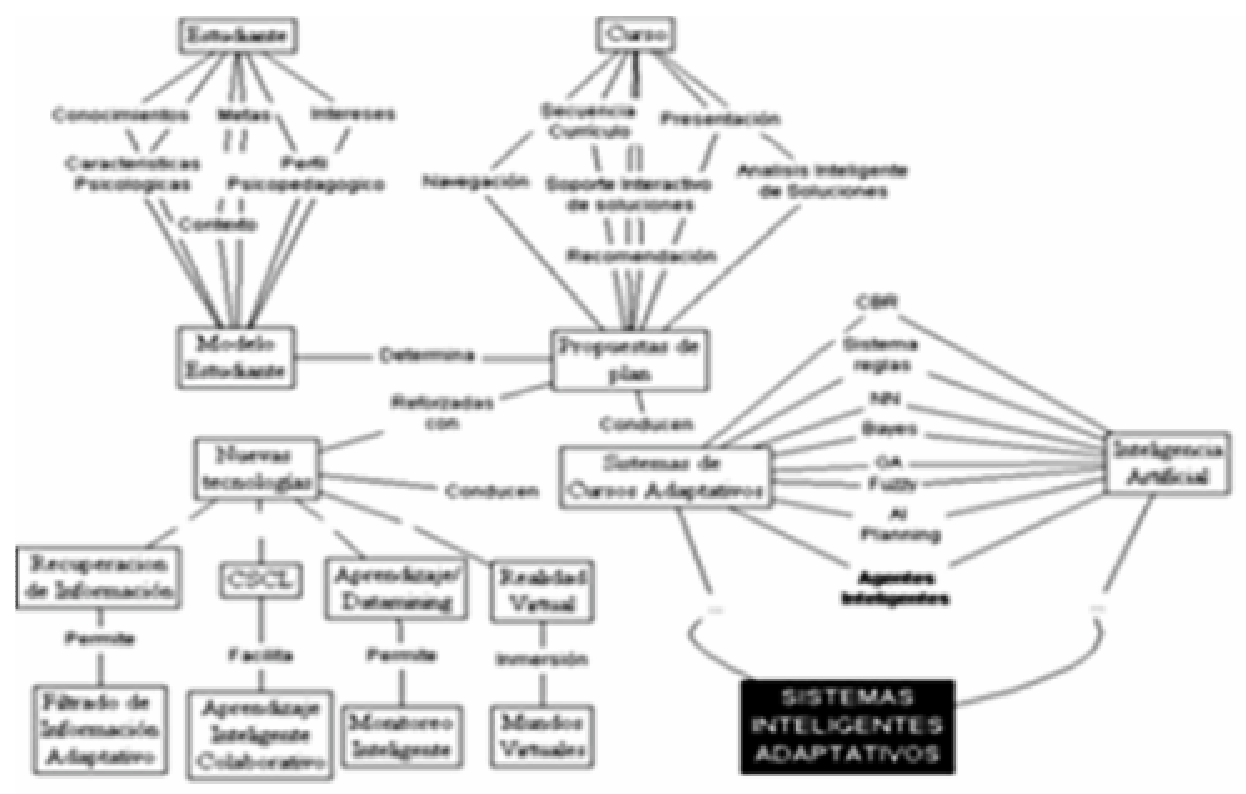

Figura 2. Mapa conceptual de propuestas en sistemas adaptativos inteligentes

La estrategia adaptativa relaciona unos elementos con otros, para generar un plan de curso inicial y mediante el monitoreo del comportamiento del estudiante en el proceso se decide si se requiere la replanificación. La estrategia de planificación y replanificación se presenta compleja por la forma de expresar el curso, por la granularidad fina de los materiales educativos (unidades educativas) y por las características heterogéneas del estudiante, pero la aplicación de diferentes técnicas inteligentes permite obviar gran parte de las dificultades sin sacrificar el objetivo. 
Tratando de atender los retos planteados por la comunidad esta propuesta se orienta hacia la definición de una estrategia genérica de adaptatividad que permita incluir diversos enfoques pedagógicos y tecnológicos, en lo referente al perfil de estudiante, la organización y especificación de los recursos pedagógicos (entendidos como cualquier objeto material o inmaterial, natural o artificial que pueda ser usado para crear o desarrollar competencias) y los componentes a adaptar en la personalización del curso. Esto genera nuevos desafíos y sin duda hace más compleja la labor.

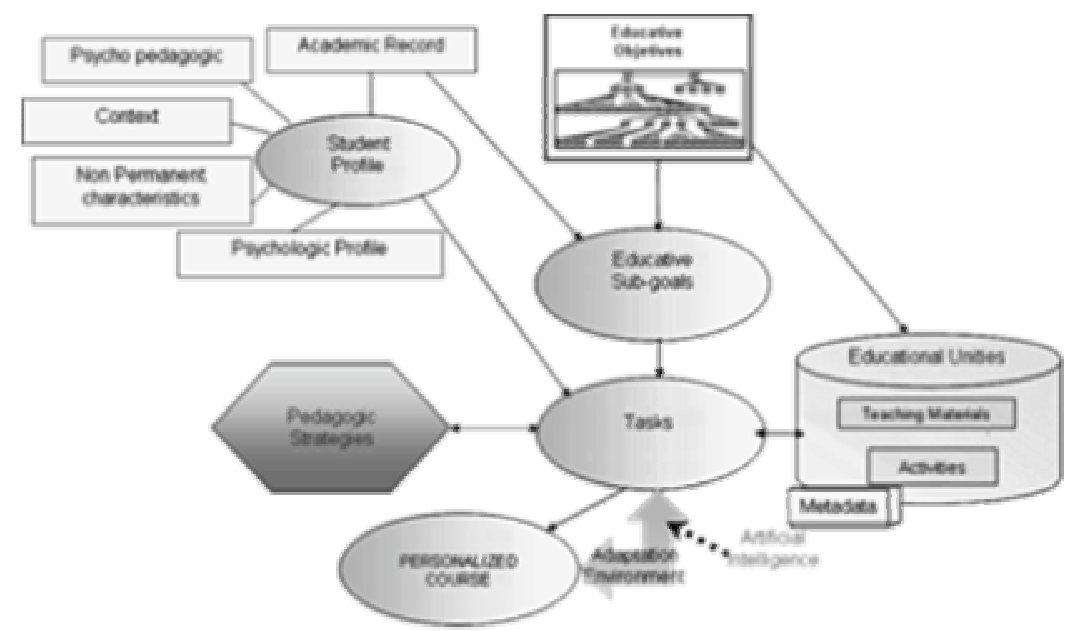

Figura 3. Generación de curso personalizado (Duque et al., 2006).

El camino a tomar puede orientarse por la descomposición en bloques funcionales, sin perder la visión sistémica, y esto conlleva a distribuir la solución en diversas entidades que requieren conocimiento específico, procesamiento y comunicación entre ellas. Ante estas características, modelar el problema mediante un Sistema Multiagente (SMA) se ve prometedor. La motivación principal para optar por un SMA está en la posibilidad de distribuir los componentes de inteligencia enmarcados en la solución al problema propuesto. Esto repercute directamente en el desarrollo modular del sistema, lo que a la vez facilita la refinación o intercambio, según el enfoque del implementador, de cada uno de los aspectos sin afectar los demás sustancialmente. Como soporte a estas expectativas el referencial teórico muestra que en la solución a problemas como el planteado, basados en conocimiento, se ha conseguido muy buenos resultados y se espera aprovechar estas posibilidades.

\section{Diseño y construcción del SMA}

A partir de esta decisión, se inicia el proceso de diseño del sistema utilizando como base la metodología MASCommonKADS la cual define los modelos necesarios para las fases de análisis y diseño y provee una completa documentación, además las aplicaciones en casos similares reportan muy buenos resultados (Iglesias, 1998). Sin embargo la experiencia ganada sugiere incluir algunos modelos de las metodologías MaSE (Multiagent system Software Engineering) (DeLoach, 2005) (Diagramas de jerárquicos de objetivos, como apoyo en la división de algunas tareas complejas) y de la metodología GAIA (Wooldridge et al., 2000) (modelo de roles, importante para determinar claramente lo esperado de los agentes).

La figura 4 recoge algunos ejemplos de los modelos concebidos. 
Para la descripción de las tareas que requieren conocimiento se especifica el control que determina cómo se combinan las inferencias elementales para conseguir un objetivo.

La descripción de las conversaciones entre los agentes se realiza en forma gráfica y textual. Estas últimas se esquematizan en plantillas como se aprecia en la figura donde participan el agente estudiante y el agente de adaptación.

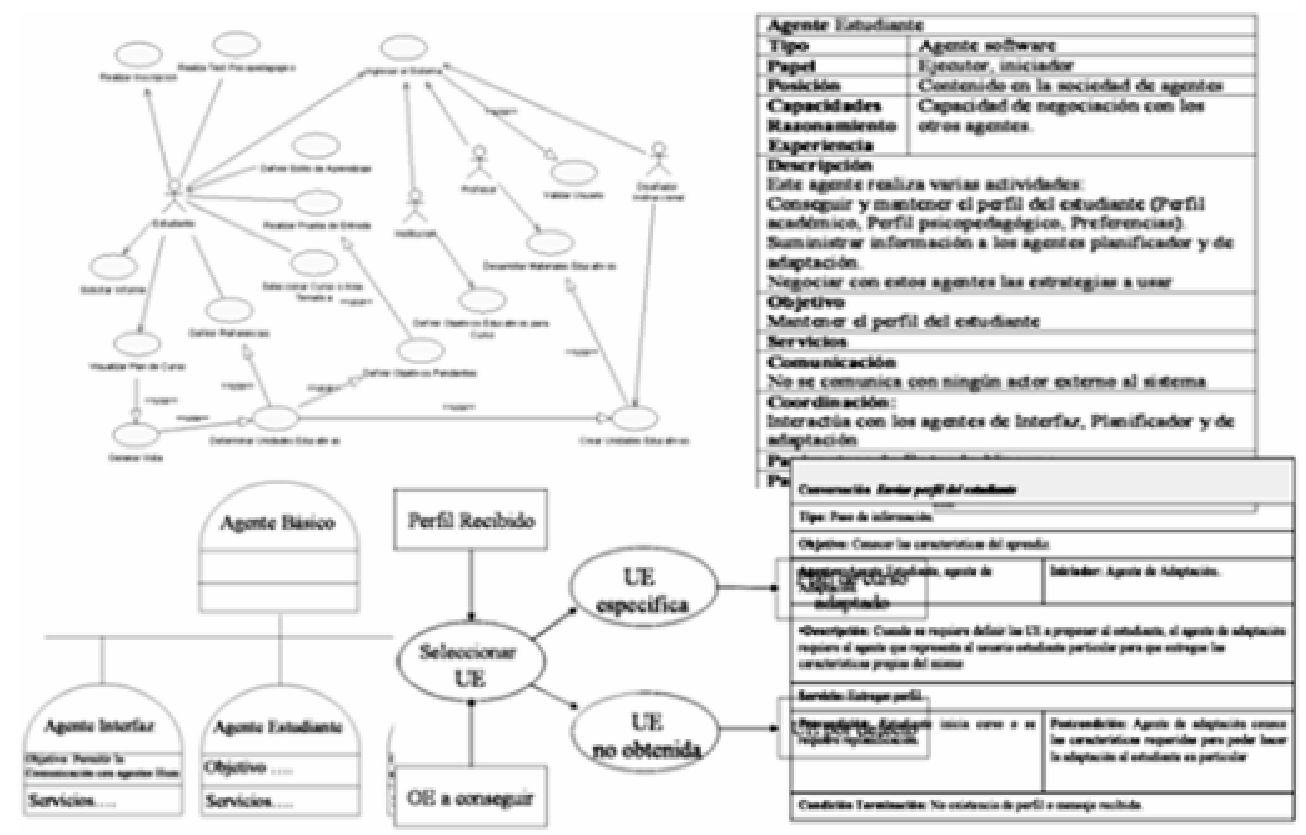

Figura 4. Diagramas de modelos utilizados en el desarrollo del SMA

Un diagrama completo del sistema propuesto se entrega en la figura 5.

El Agente Estudiante permite manejar diversos elementos en el perfil del aprendiz según el interés particular. Para la captura se utilizan formularios y test provistos por los promotores de las características incluidas, tales como pruebas psicológicas, test de estilos de aprendizaje, sociogramas y algunos valores obtenidos directamente del sistema en el proceso de interacción. Para la actualización se plantean dos formas: a) nuevos tests o formularios, b) el seguimiento de las acciones del estudiante y en algunos casos mediante aprendizaje utilizando técnicas de minería de datos sobre logs y datos recolectados.

El Agente de Dominio administra la estructura del curso asociada a un grafo acíclico cuyos nodos son los objetivos educativos (OE) a obtener, a la vez que mantiene información de las unidades educativas o recursos pedagógicos (UE).

La recuperación de los objetos o elementos almacenados se encarga al Agente de Recuperación Local que únicamente conoce la forma en que encontrará los recursos, permitiendo que se utilicen diversas visiones en la composición, nombramiento y localización de recursos, pero apoyando las tecnologías estándares en particular de los objetos de aprendizaje como LOM (IEEE, 2002) y DCMI.

Existe un agente encargado de los procesos de evaluación, realización de tests, pruebas de entrada, etc. que posee el conocimiento necesario para clasificar y ubicar al estudiante.

Como se aprecia la estrategia de adaptación está embebida en el Agente Planificador que internamente es un sistema de planificación artificial de red jerárquico de tareas 
(HTN) que permite generar el curso personalizado (Nau, 2003; Erol, 1995). Llevar el proceso de generación del curso personalizado a un problema de planificación implica definir la tripleta (S, T, D); donde $\mathrm{S}$ es el estado inicial del estudiante (académico, psicopedagógico, etc.) descrito en el lenguaje del planificador; $\mathrm{T}$ es la lista de tareas a lograr para obtener los resultados esperados; y D representa el dominio del planificador compuesto por operadores $(\mathrm{O})$ que definen las acciones directas que se pueden ejecutar y son de la forma ( $\mathrm{h}(\mathrm{v})$ Pre Del Add), y los métodos (M)que representan macro operadores que permiten "empaquetar" una lista de acciones y son de la forma (h(v) Pre $\mathrm{T})$, o de manera más general como $(\mathrm{h}(\mathrm{v})$ Pre1 T1 Pre2 T2 . . Pren Tn).

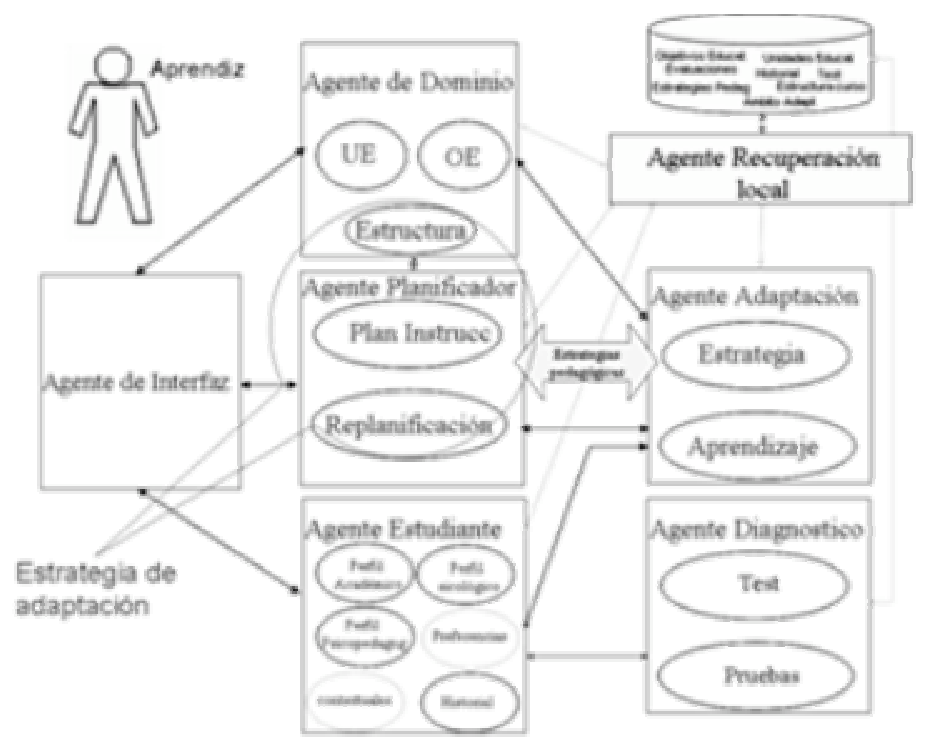

Figura 5. Diagrama del Sistema Multiagente propuesto

Para este proceso de traducción se definieron los algoritmos necesarios, presentados en (Duque et al., 2005) y de los cuales se obtiene:

$O=(U E . i d($ UE.prereq) $(\phi)(h(U E . i d))(U E . s i z e))$

Donde:

$U E . i d$ identificador de la $U E$,

UE.prereq: Pre-requisito de $U E$,

UE.size Tamaño o costo de la Unidad Educativa (Esta variable podría ser reemplazada por tiempo típico de instrucción u otra similar) y

$h(U E . i d)$ es un procedimiento que retorna los $O E$ que apoya la $U E$.

$$
M=(O E . i d(f(O E . i d))(g(O E . i d)))
$$

Donde:

$O E . i d$ : identificador del $O E$,

$f(O E . i d)$ es un procedimiento que retorna $O E \_P R E . p r e r e q$, $g(O E . i d)$ es un procedimiento que retorna Sub_OEn, OE.id

$\left(S u b \_O E_{1}, S u b \_O E_{2}, \ldots, S u b \_O E_{n}\right)$ son Objetivos educativos hijos o sub-objetivos

$\mathrm{Y}$

$$
M=\left(O E . i d\left(\left(U E_{1} . L S U E_{1} . i d\right)\left(U E_{2} . L S U E_{2} . i d\right) \ldots\left(U E_{n} . L S U E_{n} . i d\right)\right)\right.
$$

Donde: 
$O E . i d$, identificador del $O E$

$L S_{n} .:$ Estilo de aprendizaje asociado a la $U E_{n}$

El algoritmo de planificación permite ir desde el estado inicial e ir cubriendo las tareas requeridas según $\mathrm{T}$ mediante la aplicación de los métodos y operadores en $\mathrm{D}$ que soportan la "estrategia" declarada. Al final el plan personalizado se despliega como una secuencia de actividades soportadas por las Unidades Educativas (operadores) que deben ser desarrolladas por el estudiante para lograr los objetivos propuestos en el curso.

En el caso que la ejecución del plan no produzca los resultados esperados o el aprendiz encuentre dificultades en el logro de los objetivos educativos, se requiere la replanificación del curso en forma local o global. Como una buena y sencilla alternativa se opta por la técnica de inteligencia artificial conocida como Razonamiento Basado en Casos (RBC). El principio básico en RBC es que problemas similares tienen soluciones similares, lo que permite reusar las soluciones exitosas ante problemas similares. Para el caso particular implica recuperar la información que el sistema ha obtenido de las experiencias exitosas de planes para atender los objetivos propuestos en estudiantes con perfil similar.

La plataforma multiagente fue construida en java con el apoyo para el desarrollo del reconocido Framework JADE (Jade) y los datos son almacenados en MySQL. El sistema es un desarrollo Web, con base en herramientas libres y multiplataforma, por lo cual se puede instalar los servidores tanto en ambientes Windows como Linux y permite el acceso desde cualquier plataforma que posea un navegador Web

\section{Conclusiones y trabajos futuros}

Enfrentar las deficiencias encontradas en el aprovechamiento de las nuevas tecnologías en los sistemas de educación virtual, en particular la falta de personalización de los cursos, pasa por definir los elementos que deben ser tenidos en cuenta en cada estudiante para adaptar el curso y a la vez asociar estas diferencias con materiales y actividades que reconozcan, en la práctica, a cada estudiante, buscando su satisfacción académica y espiritual, generando motivación y proyectando mecanismos metacognitivos que en últimas se traducen en una mejor efectividad del proceso.

Tiene gran importancia la definición de la estructura del curso y la composición a partir de elementos de alta granularidad, que permitan una detallada adaptación. Estas ventajas generan problemas en la construcción y gestión manual y las técnicas empleadas en la solución juegan su rol específico, en particular los agentes inteligentes, la planificación en inteligencia artificial y el razonamiento basado en casos.

La plataforma está en mejoramiento y sobre la misma se adelantan nuevos proyectos que permitan mejorar la aplicación, en particular afinar el proceso de ejecución y la implementación de los módulos de evaluación e interfaces adaptativas.

\section{Referencias}

ALFARO, L., PEREIRA, F. JACINTHO, L. Modelagem de um Ambiente Inteligente para a Educação baseado em Realidade Virtual. Memorias IV Congresso RIBIE, Brasilia, 1998.

BRUSILOVSKY, P, MAYBURY, M. From Adaptive Hypermedia to the Adaptive Web, Communication of the ACM. Vol 45 No. 5, 2002. 
CRISTEA, A. CARRO, R. GARZOTTO, F. Presentation of A3EH: The 3rd workshop of Authoring of Adaptive and Adaptable Educational Hypermedia. AIED. Holanda. 2005.

DASTBAZ, M., MUSTAFA, A, STONEHAM, R. Issues in Design and Development of Personalised E-Learning Systems. Memorias. ED-MEDIA 2006. World Conference on Educational Multimedia, Hypermedia \& Telecomunications. Orlando. USA. 2006.

DCMI. The Dublin Core Metadata Initiative DCMI. DCMI Recommendations. Disponible en: http://dublincore.org/index.shtml

DELOACH, S. Multiagent Systems Engineering Of Organization-Based Multiagent Systems. Memorias 4th Intenacional Workshop On Software Engineering For LargeScale Multi-Agents Systems Selmas'05, St. Louis, 2005.

DUQUE, N. Modelo adaptativo multi-agente para la Planificación y ejecución de cursos Virtuales personalizados. Propuesta de Tesis de Doctorado en IngenieríaSistemas. Universidad Nacional de Colombia - Sede Medellín, pp. 34, 2007.

DUQUE, N OVALLE, D. JIMÉNEZ, J. Artificial intelligence for automatic generation of Customized courses. Memorias. ED-MEDIA 2006-World Conference on Educational Multimedia, Hypermedia \& Telecommunications, USA, 2006.

DUQUE, N. JIMÉNEZ, C. GUZMAN, J. AI Planning for Automatic Generation of Customized Virtual Courses, Frontiers in Artificial Intelligence and Applications. IOS Press, Vol 117. Ámsterdam, 2005.

ED-MEDIA 2006. Memorias World Conference on Educational Multimedia, Hypermedia \& Telecomunications. Orlando. USA. 2006.

ERAUT, S. Un método de sistemas instructivos en el desarrollo del curso. Psicología de la educación, recopilación E. Stones, Tomo II, Ediciones Morata, Madrid, 1970.

EROL, K. Hierarchical Tasks Network Planning: Formalization, analysis and Implementation. Dissertation Doctor of Philosophy. 1995.

IEEE. Learning Technology Standards Committee. Learning Object Metadata Standard Maintenance/Revision. 2002.

IGLESIAS, C.. Definición de una metodología para el desarrollo de sistemas multiagente. Tesis doctoral. Departamento de ingeniería de sistemas telemáticos. Universidad Politécnica de Madrid. 1998.

ITS 2006. Memorias. 8th International Conference Intelligents Tutoring Systems. Taiwán. 2006.

JADE. Java Agent DEvelopment framework. Departamento de Ingeniería de la Información de la Universidad de Parma. Guias. Disponible en: http://jade.tilab.com. 
JAQUES, P. VICARI, R. PAT: Um agente pedagógico animado para interagir afetivamente com o aluno. Brasil. 2005.

JIMÉNEZ, J. Un Modelo de Planificación Instruccional usando Razonamiento Basado en Casos en Sistemas MultiAgente para entornos integrados de Sistemas Tutoriales Inteligentes y Ambientes Colaborativos de Aprendizaje Apoyados en Computador. Tesis doctoral. Colombia. 2006.

KARAGIANNIDIS, C. KOUMPIS, A. STEPHANIDIS, C. Deciding 'What', 'When', 'Why', and 'How' to Adapt in Intelligent Multimedia Presentation Systems. Memorias. 12th European Conference on Artificial Intelligence, Budapest, Hungary. 1996.

MARTINS, W. DINIZ DE CARVALHO, An intelligent tutoring system based on selforganizing maps- design, implementation and evaluation. Memorias ITS 2004. Brasil.

MERIDA, D. FABREGAT, R. SHAAD: Sistema Hipermedia Adaptable, Adaptativo y Dinámico para entrega de Contenidos Hipermedia. Universidad de Girona. 2003.

NAKAGAWA, M. KURODA, T. A Case-Based Reasoning System on the Internet for References of Information Technology Education to Teachers. Proceedings of the International Workshop on Current Trends and Applications of Artificial Intelligence in Education, Ayala, G. 2004.

NAU, D. TSZ-CHIU, A. KUTER, U. MURDOCK, W. WU, D. YAMAN, F. SHOP2: An HTN Planning System, Journal of Artificial Intelligence Research. Disponible www.cs.cmu.edu/afs/cs/project/jair/pub/volume20/nau03a.pdf. 2003.

PAQUES, H LIU, L AND PU, C Adaptation Space: A Design Framework for Adaptive Web Services. Georgia Institute of Technology, USA. 2004.

SILVEIRA, R. Modelagem Orientada a Agentes Aplicada a Ambientes Inteligentes Distribuídos de Ensino: JADE Java Agent framework for Distance learning Environments. Universidade Federal do Rio Grande do Sul. Tesis Doctoral. 2001.

STOLUROW, L. S. C.A.I. Algunos problemas y perspectivas. Psicología de la educación, a cargo de E. Stones, Tomo II. Ediciones Morata. Madrid. 1970.

VICARI, R. Agentes Inteligentes en Educación Virtual. Memorias. Seminario Internacional. Inteligencia Artificial y Seguridad. Pereira, Colombia. 2005.

WOOLDRIDGE M., N. J., D. KINNY. The Gaia Methodology For Agent-Oriented Analysis And Design. International Journal Of Autonomous Agents And MultiAgent Systems, 2000. 